\title{
HOMOLOGY AND COHOMOLOGY GROUPS OF COMMUTATIVE BANACH ALGEBRAS AND ANALYTIC POLYDISCS
}

\author{
L. I. PUGACH and M. C. WHITE \\ Department of Mathematics, The University, Newcastle upon Tyne NE1 7RU, England
}

(Received 23 February, 1998)

\begin{abstract}
In this paper we deduce the existence of analytic structure in a neighbourhood of a maximal ideal $M$ in the spectrum of a commutative Banach algebra, $A$, from homological assumptions. We assume properties of certain of the cohomology groups $H^{n}(A, A / M)$, rather than the stronger conditions on the homological dimension of the maximal ideal the first author has considered in previous papers. The conclusion is correspondingly weaker: in the previous work one deduces the existence of a Gel'fand neighbourhood with analytic structure, here we deduce only the existence of a metric neighbourhood with analytic structure. The main method is to consider products of certain co-cycles to deduce facts about the symmetric second cohomology, which is known to be related to the deformation theory of algebras.
\end{abstract}

1991 Mathematics Subject Classification. 46J20, 46M20

1. Introduction. This work can be considered as one in the series of papers by the first author which study homological properties of non-idempotent maximal ideals in commutative Banach algebras ([8], [9]). From another point of view it continues the long series of paper by many authors which studied conditions on the vanishing of cohomology groups of Banach algebras.

Throughout this paper $A$ denotes a commutative Banach algebra with unit 1 , $M \subset A$ is a fixed maximal ideal. We denote by $\mathcal{M}_{A}$ the space of maximal ideals of $A$ and consider two topologies on $\mathcal{M}_{A}$ - the Gelfand topology and norm (metric) topology, induced by the norm from $A^{*}$. The letter $\omega$ stands for the point in $\mathcal{M}_{A}$, that corresponds to $M$ and for the multiplicative functional annihilating $M$.

The symbol $\hat{\otimes}$ denotes the projective tensor product of Banach spaces [4], and $\pi: M \hat{\otimes} M \rightarrow M$ is the operator of multiplication. We denote by $M^{2}$ the algebraic square of $M$ (that is the linear space generated by $a b,(a, b \in M)$ ). We also consider the topological square $\overline{M^{2}}$, which is the closure of $M^{2}$. The ideal $M$ is called idempotent if $M=\overline{M^{2}}$ and non-idempotent otherwise. Note that the quotient space $M / \overline{M^{2}}$ is the predual of the space of bounded point derivations at the point $\omega$.

There was a series of papers in the 60's and 70's (see [2], [11]) that tried to connect the behaviour of bounded point derivations at point $\omega$ (and therefore of the closed powers of the ideal $M$ ) with an analytic structure in $\mathcal{M}_{A}$. We recall that the subset $\mathcal{U} \subset \mathcal{M}_{A}$ is called an $n$-dimensional analytic polydisc if there is a homeomorphism $\gamma$ from the open unit polydisc $\mathcal{D}^{n} \subset \mathbf{C}^{n}$ onto $\mathcal{U}$ such that for all $a \in A$ the function $\hat{a} \circ \gamma$ is holomorphic in $\mathcal{D}^{n}$ (where $\hat{a}$ denotes the Gelfand transformation of $a$ ). 
Perhaps the most detailed results concerning powers of a maximal ideal and analytic structure are in [11]. We shall use a much simplified version of the main results of [11] and present it in the following theorem:

\section{THEOREM R. If}

(i) $\operatorname{dim} \overline{M^{k}} / \overline{M^{k+1}}=\left(\begin{array}{c}n+k-1 \\ k\end{array}\right)$ for all natural numbers $k$, and

(ii) $\pi(M \hat{\otimes} M)=\overline{M^{2}}$,

then there exists a neighbourhood $\mathcal{U}$ of the point $\omega$ in the metric topology that is an n-dimensional analytic polydisc.

Note that the condition ( $i$ ) says that the powers of $M$ behave in the best possible manner - as in the polydisc algebra $A\left(\mathcal{D}^{n}\right)$.

A brief outline of the proof of this theorem can be found in [10]. This theorem was a principal tool in the papers of the first author $([\mathbf{8}]-[\mathbf{1 0}])$, where the existence of an analytic polydisc was deduced from such homological conditions as projectivity, flatness and finite homological dimension of $M$ as a Banach $A$-module. Now we consider weaker homological conditions - namely the triviality of some homology and cohomology groups of the Banach algebra $A$.

We denote by $\mathbf{C}$ the trivial bimodule $A / M$ and consider the Hochschild homology group $H_{n}(A, \mathbf{C})$ and cohomology group $H^{n}(A, \mathbf{C})$ (note that these are respectively equal to $H_{n}(M, \mathbf{C})$ and $\left.H^{n}(M, \mathbf{C})\right)$. As usual we denote by $L^{n}(M, \mathbf{C})$ the space of all continuous $n$-linear maps from $M \times M \times \cdots \times M$ ( $n$ times) to $\mathbf{C}$, by $\mathcal{Z}_{n}(M, \mathbf{C})$ the group of all $n$-cycles, by $\mathcal{B}_{n}(M, \mathbf{C})$ the group of all $n$-boundaries, by $\mathcal{Z}^{n}(M, \mathbf{C})$ the group of all $n$-cocycles and by $\mathcal{B}^{n}(M, \mathbf{C})$ the group of all $n$ coboundaries [7]. We denote by $d$ the boundary operator and $\delta$ the coboundary operator (usually we omit the degree of $d$ and $\delta$ ). Note that the symbol $\delta_{i j}$ stands for the Kronecker delta.

To define the symmetric homology and cohomology groups we introduce the following operator on $n$-chains: $\left(a_{1} \otimes a_{2} \otimes \cdots \otimes a_{n}\right)^{o p}=(-1)^{\frac{1}{n} n(n+1)-1}\left(a_{n} \otimes a_{n-1}\right.$ $\left.\otimes \cdots \otimes a_{1}\right)$. The symmetric and antisymmetric chains are the +1 and -1 eigenspaces of the operator op. One easily checks that $d\left(\eta^{o p}\right)=(d \eta)^{o p}$ for all $n$-chains $\eta$. This shows that the symmetric and antisymmetric chains form two subcomplexes of the spaces of all chains. The homology of these subcomplexes are the symmetric and antisymmetric homology. In exactly the same fashion we define $\phi^{o p}(\eta)=\phi\left(\eta^{o p}\right)$ for an $n$-cochain $\phi$. Again $\delta\left(\phi^{o p}\right)=(\delta \phi)^{o p}$ and the homology of the resulting subcomplexes of eigenspaces gives the symmetric and antisymmetric cohomology. When we ornament a space of (co)-chains, (co)-cycles, (co)-boundaries or (co)-homology groups by $a$ or $s$ we mean the corresponding symmetric or antisymmetric version. Note in each case the usual space is the direct sum of the symmetric and antisymmetric versions.

We mainly consider the symmetric second cohomology group $H_{s}^{2}(M, \mathbf{C})$, which is important in the theory of extensions of Banach algebras, see [1].

2. The $\pi$-property. Theorem $\mathrm{R}$ includes the condition that the image of the canonical multiplication operator $\pi: M \hat{\otimes} M \rightarrow M$ must coincide with $\overline{M^{2}}$. Note that we always have $M^{2} \subseteq \operatorname{Im} \pi \subseteq \overline{M^{2}}$ and hence $\operatorname{Im} \pi=\overline{M^{2}}$ if and only if $\operatorname{Im} \pi$ is closed. We shall call this condition the $\pi$-property. 
Actually one often meets the $\pi$-property in Banach homology theory (see, for example [8], [10]). In particular projective and flat [8] maximal ideals have this property. All $M$ possessing a bounded approximate identity satisfy $M^{2}=M$, by Cohen's factorization theorem hence have the $\pi$-property. An unbounded approximate identity is not enough: for instance, the algebra $l^{2}(\mathbf{N})$, with pointwise operations, does not have the $\pi$-property $\left(\operatorname{Im} \pi=l^{1}(\mathbf{N})\right)$. The following result shows a relationship between the $\pi$-property and the second cohomology group.

Proposition 1. If $H_{s}^{2}(M, \mathbf{C})=0$, then $M$ has the $\pi$-property.

Proof. Assume the contrary: that $\operatorname{Im} \pi$ is not closed. In this case the usual norm on $\operatorname{Im} \pi$, that induced from $M$, does not agree with the factor-norm generated by the operator $\pi: M \hat{\otimes} M \rightarrow \operatorname{Im} \pi$. We can choose a functional $f: \operatorname{Im} \pi \rightarrow \mathbf{C}$, which is bounded in the factor norm (that is $|f(\pi(u))| \leq K\|u\|$ for all $u \in M \hat{\otimes} M$ ), but unbounded in the usual norm. Consider the functional $\psi=f \circ \pi$ on $M \hat{\otimes} M$. Since $\psi$ is bounded and $\psi(a b \otimes c)=\psi(a \otimes b c)$, it generates a cocycle in $\mathcal{Z}^{2}(M, \mathbf{C})$, which is obviously symmetrical. This implies that $\psi=g \circ \pi, g \in M^{*}$, and hence the bounded $g$ and unbounded $f$ agree on the dense subset $\operatorname{Im} \pi$ of $\overline{M^{2}}$. This contradiction proves the result.

COROLlary. If $H^{2}(M, \mathbf{C})=0$, then $M$ has the $\pi$-property.

Proof. If every 2-cocycle is a coboundary, then every symmetrical 2-cocycle is a 2-coboundary and the result follows from Proposition 1.

The following result is far more general than Proposition 1 and its Corollary.

Theorem 1. If $H^{n+2}(M, \mathbf{C})=0$ and $\operatorname{dim} M / \overline{M^{2}}=m \geq n \geq 0$, then $M$ has the $\pi$ property.

Proof. If $\operatorname{Im} \pi$ is not closed, then there exists $f: \operatorname{Im} \pi \rightarrow \mathbf{C}$, which is bounded in the factor-norm, but unbounded in the usual norm of $M$. Since $\operatorname{dim} M / \overline{M^{2}}=$ $m \geq n \geq 0$ we can choose $h_{1}, \ldots, h_{n} \in M$ and $t_{1}, \ldots, t_{n} \in M^{*}$ such that $t_{i}\left(h_{j}\right)=\delta_{i j}$ and each $t_{j}$ annihilates $\overline{M^{2}}$. Now consider $T \in L^{n+2}(M, \mathbf{C})$, given by the formula

$$
T\left(a_{1}, \ldots, a_{n+2}\right)=t_{1}\left(a_{1}\right) \ldots t_{n}\left(a_{n}\right) f\left(a_{n+1} a_{n+2}\right) .
$$

It is obvious that $\delta T=0$ and so $T \in \mathcal{Z}^{n+2}(M, \mathbf{C})$. Hence $T \in \mathcal{B}^{n+2}(M, \mathbf{C})$ and so there exists $S \in L^{n+1}(M, \mathbf{C})$ such that $T=\delta S$. This means that

$$
\begin{aligned}
t_{1}\left(a_{1}\right) \ldots t_{n}\left(a_{n}\right) f\left(a_{n+1} a_{n+2}\right)= & S\left(a_{1} a_{2}, a_{3}, \ldots, a_{n+2}\right)-S\left(a_{1}, a_{2} a_{3}, \ldots, a_{n+2}\right)+\cdots \\
& +(-1)^{n} S\left(a_{1}, a_{2}, \ldots, a_{n}, a_{n+1} a_{n+2}\right) .
\end{aligned}
$$

Now fix $a, b \in M$ and substitute in (*) instead of $a_{1}, a_{2}, \ldots, a_{n+2}$ all permutations of $h_{1}, h_{2}, \ldots, h_{n}, a, b$ such that $a$ is to the left of $b$. Every time we multiply the equality we obtain by the sign of the corresponding permutation. Then we add all the equations we have obtained. The only remaining term on the left-hand side is $f(a b)$, because the 
other terms were either zero or have cancelled in pairs. On the right-hand side the summands containing $a b$ appear exactly once, while the other summands will appear twice with different signs and cancel pairwise. So we obtain the following equality,

$$
\begin{aligned}
f(a b) & =S\left(a b, h_{1}, \ldots, h_{n}\right)-S\left(h_{1}, a b, \ldots, h_{n}\right)+\cdots \\
& +(-1)^{n+1} S\left(h_{n}, h_{n-1}, \ldots, h_{1}, a b\right) .
\end{aligned}
$$

which has $(n+1)$ ! summands on the right-hand side. This means that $f$ is bounded on $\overline{M^{2}}$ and therefore on $M$. This contradiction finishes the proof.

The following result shows that the tensor product of Banach algebras in some sense preserves the $\pi$-property.

Proposition. Let $A_{1}$ and $A_{2}$ be commutative Banach algebras with unit. Let $M_{i}$ be an idempotent maximal ideal in $A_{i}$ which has the $\pi$-property $(i=1,2)$. Then the corresponding maximal ideal $M$ in $A_{1} \hat{\otimes} A_{2}$ also has the $\pi$-property.

Proof. We have $\pi_{i}\left(M_{i} \hat{\otimes} M_{i}\right)=M_{i},(i=1,2)$. Using the well-known fact [4] that arbitrary $u \in M_{1} \hat{\otimes} M_{2}$ can be represented in the form $u=\sum_{k=1}^{\infty} a_{k} \otimes b_{k}, a_{k} \in M_{1}$, $b_{k} \in M_{2}$ and $\sum_{k=1}^{\infty}\left\|a_{k}\right\|\left\|b_{k}\right\|<\infty$ and the open mapping theorem, we easily see that there exists $U \in M_{1} \hat{\otimes} M_{1} \hat{\otimes} M_{2} \hat{\otimes} M_{2}$ such that $\left(\pi_{1} \otimes \pi_{2}\right) U=u$. Now we take an arbitrary element $v \in M \subset A_{1} \hat{\otimes} A_{2}$ and represent it in the form $v=u+m_{1} \otimes 1_{2}$ $+1_{1} \otimes m_{2}$, where $u \in M_{1} \hat{\otimes} M_{2}, m_{i} \in M_{i}, 1_{i}$ is the unit of $A_{i}(i=1,2)$.

Consider the linear operator $P: A_{1} \hat{\otimes} A_{1} \hat{\otimes} A_{2} \hat{\otimes} A_{2} \rightarrow A_{1} \hat{\otimes} A_{2} \hat{\otimes} A_{1} \hat{\otimes} A_{2}$ given by $P(a \otimes b \otimes c \otimes d)=a \otimes c \otimes b \otimes d$. Note that $\left.\pi_{M} \circ P\right|_{M_{1} \otimes M_{1} \otimes M_{2} \otimes M_{2}}=\pi_{1} \otimes \pi_{2}$. Then take the chosen element $U \in M_{1} \hat{\otimes} M_{1} \hat{\otimes} M_{2} \hat{\otimes} M_{2}$ such that $\left(\pi_{1} \otimes \pi_{2}\right) U=u$ and choose elements $u_{i} \in M_{i} \hat{\otimes} M_{i}$ such that $\pi_{i}\left(u_{i}\right)=m_{i},(i=1,2)$.

Now consider $V=P\left(U+u_{1} \otimes 1_{2} \otimes 1_{2}+1_{1} \otimes 1_{1} \otimes u_{2}\right) \in A_{1} \hat{\otimes} A_{2} \hat{\otimes} A_{1} \hat{\otimes} A_{2}$. First we can easily check that $V \in M \hat{\otimes} M$ : it is enough to verify that $(\omega \otimes 1) V,(1 \otimes \omega) V$ and $(\omega \otimes \omega) V$ are all equal to zero, where $\omega$ is the multiplicative functional annihilating $M$.

After that the calculation $\pi_{M}(V)=\pi_{M} P\left(U+u_{1} \otimes 1_{2} \otimes 1_{2}+1_{1} \otimes 1_{1} \otimes u_{2}\right)=$ $\left(\pi_{1} \otimes \pi_{2}\right)\left(U+u_{1} \otimes 1_{2} \otimes 1_{2}+1_{1} \otimes 1_{1} \otimes u_{2}\right)=u+\pi_{1}\left(u_{1}\right) \otimes 1_{2}+1_{1} \otimes \pi_{2}\left(u_{2}\right)=u+m_{1}$ $\otimes 1_{2}+1_{1} \otimes m_{2}=v$ proves that $\pi_{M}(M \hat{\otimes} M)=M$.

Note. The $\pi$-property is very close to the well-known $S$-property (see [6]), which is equivalent to the coincidence of the following two norms on $M^{2}$ : the usual norm (from $M$ ) and the factor-norm given by the operator of multiplication $\pi_{\alpha}: M \otimes M \rightarrow M$. It is easy to see that the $S$-property always implies the $\pi$-property. The example due to P.Dixon [3] shows that the converse implication is not true: there exists a Banach algebra $A_{0}$ such that $A_{0}^{2}=A_{0}$ (and hence $A_{0}$ has the $\pi$-property) but $A_{0}$ does not have the $S$-property. In this example $A_{0}$ is neither commutative nor semisimple. It would be interesting to know whether the $\pi$-property and $S$ property are equivalent in each of these cases.

3. The powers of a maximal ideal. Now we establish a sufficient condition for the powers of the given maximal ideal $M \subset A$ to behave in the best possible manner, that is $\operatorname{dim} \overline{M^{k}} / \overline{M^{k+1}}=\left(\begin{array}{c}n+k-1 \\ k\end{array}\right)$ for all natural numbers $k$. 
Proposition 2. If $M$ has the $\pi$-property and the functional $G_{\mu}$, whenever it is defined, annihilates the kernel of the multiplication operator $\pi$, then $\operatorname{dim} \overline{M^{k}} / \overline{M^{k+1}}=\left(\begin{array}{c}n+k-1 \\ k\end{array}\right)$ for all natural numbers $k$.

Proof. We suppose that $\operatorname{dim} M / \overline{M^{2}}=n$ and choose $h_{1}, \ldots, h_{n} \in M$ and $t_{1}, \ldots, t_{n} \in M^{*}$ such that $t_{i}\left(h_{j}\right)=\delta_{i j}$ and each $t_{i}$ annihilates $\overline{M^{2}}$.

We denote by $\Lambda$ the set of all multi-indices of length $n: \lambda=\left(\kappa_{1}, \ldots, \kappa_{n}\right)$ and $|\lambda|=\kappa_{1}+\cdots+\kappa_{n}$ the power of $\lambda$. Denote also $h^{\lambda}=h_{1}^{\kappa_{1}} \ldots h_{n}^{\kappa_{n}}$ and $e_{k}=(0, \ldots$, $1, \ldots, 0)$ where the unit is in the $k$-th place.

We shall construct, by induction on $m=|\lambda|$, a collection of functionals $t_{\lambda}$, $\lambda \in \Lambda$, such that $t_{\lambda}$ annihilates $\overline{M^{m+1}}$ and where $t_{\lambda}\left(h^{\nu}\right)=\delta_{\lambda \nu}$. For $m=1$ we already have such functionals: set $t_{e_{k}}$ to be $t_{k}$, and $t_{0}=\omega$. Fix $\mu \in \Lambda,|\mu|=m+1$. Consider a formal equality $G_{\mu}=\sum_{\substack{\lambda+\nu=\mu \\|\lambda|,|v|>0}} t_{\lambda} \otimes t_{\nu}$. Provided all $t_{\lambda},|\lambda| \leq m$, already exist, it defines a functional $G_{\mu} \in(M \hat{\otimes} M)^{*}$. Note that $t_{0}=\omega$ is zero on $M$, so regardless of the subsequent choice of $t_{\mu}$ this sum will be equal to the same sum without the restriction of $|\lambda|,|\nu|>0$.

For all $\lambda \in \Lambda,|\lambda|=m$ and $t_{\lambda}$ already is defined. Now for an arbitrary fixed $\mu$, with $|\mu|=m+1$, the functional $G_{\mu}$ is defined as above. Because $G_{\mu}$ annihilates $\operatorname{Ker} \pi$, we can see from the following diagram:

$$
\begin{array}{ccc}
M \hat{\otimes} M & \\
G_{\mu} \downarrow & \searrow \\
\mathbf{C} & \stackrel{\pi}{t_{\mu}} \overline{M^{2}}
\end{array}
$$

that there exists a continuous linear functional $t_{\mu}: \overline{M^{2}} \rightarrow \mathbf{C}$ such that $t_{\mu} \circ \pi=G_{\mu}$. Then extend $t_{\mu}$ to $M$ by the formula $t_{\mu}\left(h_{k}\right)=0$ for $k=1,2, \ldots, n$. Then for arbitrary $a \in M, \quad b \in \overline{M^{m+1}} \quad$ we have $t_{\mu}(a b)=t_{\mu} \pi(a \otimes b)=G_{\mu}(a \otimes b)=$ $\sum_{\lambda+v=\mu} t_{\lambda}(a) t_{\nu}(b)=0$, because $t_{\nu}$ annihilates $\overline{M^{m+1}}$. It follows by linearity and continuity that $t_{\mu}$ annihilates $\overline{M^{m+2}}$. The same type of argument shows that $t_{\mu}\left(h^{\nu}\right)=\delta_{\mu \nu}$.

It follows by induction that the system of $t_{\mu}$ exists for all $\mu \in \Lambda$. Hence the system of $h^{\mu},|\mu|=k$, has independent images in $\overline{M^{k}} / \overline{M^{k+1}}$, and thus $\operatorname{dim} \overline{M^{k}} / \overline{M^{k+1}}=\left(\begin{array}{c}n+k-1 \\ k\end{array}\right)$, and the proposition is proved.

In what follows we shall use the following property of the $t_{\gamma}$ :

LEMMA 1. $t_{\gamma}(a b)=\sum_{\alpha+\beta=\gamma} t_{\alpha}(a) t_{\beta}(b)$.

Proof. The left-hand side is equal to $t_{\gamma} \pi(a \otimes b)$, and the right-hand side is $\sum_{\alpha+\beta=\gamma} t_{\alpha} \otimes t_{\beta}(a \otimes b)=G_{\gamma}(a \otimes b)$, which is the same thing.

4. Cohomology groups and analytic polydiscs Now we shall apply our main tool, Proposition 2, to different situations and obtain the results about the existence of analytic polydiscs.

Theorem 2. Let $H_{s}^{2}(M, \mathbf{C})=0$ and $\operatorname{dim} M / \overline{M^{2}}=n$. Then there exists a neighbourhood $\mathcal{U}$ of the corresponding point $\omega \in \mathcal{M}_{A}$ in the metric topology that is an n-dimensional analytic polydisc. 
Proof. For every $\mu \in \Lambda$ the functional $G_{\mu}$ is defined. It is obvious from the definition that $G_{\mu}$ is symmetric: $G_{\mu}(a \otimes b)=G_{\mu}(b \otimes a)$. Now using Lemma 1, we can prove that $G_{\mu}(a b \otimes c)=G_{\mu}(a \otimes b c)$. In fact, $G_{\mu}(a b \otimes c)=\sum_{\alpha+\beta=\mu} t_{\alpha}(a b) \otimes t_{\beta}(c)$ $=\sum_{\alpha+\beta=\mu}\left(\sum_{\epsilon+\gamma=\alpha} t_{\epsilon}(a) t_{\gamma}(b)\right) t_{\beta}(c)=\sum_{\epsilon+\gamma+\beta=\mu} t_{\epsilon}(a) t_{\gamma}(b) t_{\beta}(c)$, and similar calculations show this is also the value of $G_{\mu}(a \otimes b c)$.

Hence the bilinear functional $g \in L^{2}(M, \mathbf{C})$, given by the formula $g(a, b)=G_{\mu}(a \otimes b)$, is a symmetric 2-cocycle. Since $H_{s}^{2}(M, \mathbf{C})=0, g \in \mathcal{B}^{2}(M, \mathbf{C})$. So $G_{\mu}(a \otimes b)=f(a b)$ for some $f \in M^{*}$, and now it is evident that $G_{\mu}$ annihilates Ker $\pi$.

Using Propositions 1 and 2 and Theorem R, we complete the proof.

Note. Certainly the requirement of symmetry of the cohomology group $H^{2}(M, \mathbf{C})$ can be omitted. But the vanishing of the usual group restricts the dimension of the linear space $M / \overline{M^{2}}$, and hence the cases to which we could apply the result.

Proposition 3. If $H^{n+1}(M, \mathbf{C})=0$, then $\operatorname{dim} M / \overline{M^{2}} \leq n$.

Proof. Assume the contrary: $\operatorname{dim} M / \overline{M^{2}} \geq n+1$. Then there exist $h_{1}, \ldots$, $h_{n+1} \in M$ and $t_{1}, \ldots, t_{n+1} \in M^{*}$ such that $t_{i}\left(h_{j}\right)=\delta_{i j}$ and each $t_{i}$ annihilates $\overline{M^{2}}$. Consider $S \in L^{n+2}(M, \mathbf{C})$ defined by the formula

$$
S\left(a_{1}, \ldots, a_{n+1}\right)=t_{1}\left(a_{1}\right) \ldots t_{n+1}\left(a_{n+1}\right) .
$$

Obviously $\delta S=0$ and hence $S \in \mathcal{Z}^{n+1}(M, \mathbf{C})$. Since $H^{n+1}(M, \mathbf{C})=0$, $S \in \mathcal{B}^{n+1}(M, \mathbf{C})$, that is $S=\delta R$ for some $R \in L^{n}(M, \mathbf{C})$. In particular, it means that

$$
\begin{aligned}
S\left(h_{1}, \ldots, h_{n+1}\right) & =R\left(h_{1} h_{2}, h_{3}, \ldots, h_{n+1}\right)-R\left(h_{1}, h_{2} h_{3}, \ldots, h_{n+1}\right)+\cdots \\
& +(-1)^{n+1} R\left(h_{1}, h_{2}, \ldots, h_{n} h_{n+1}\right) .
\end{aligned}
$$

Now consider all possible permutations of $h_{1}, \ldots, h_{n+1}$ and write down the $(n+1)$ ! corresponding equations. After addition of all these equalities multiplied by the sign of the corresponding permutation we get the number 1 on the left hand side, and on the right-hand side all the terms cancel pairwise and we get zero. This contradiction proves the result.

We obtain only a 1-dimensional result about the usual cohomology of order 2, as our maximal ideals are required to be non-idempotent.

Theorem $2^{\prime}$. If $H^{2}(M, \mathbf{C})=0$ and $M \neq \overline{M^{2}}$, then $\operatorname{dim} M / \overline{M^{2}}=1$ and there exists a metric neighbourhood $\mathcal{U}$ of the corresponding point $\omega \in \mathcal{M}_{A}$ which is a 1-dimensional analytic disc.

Now consider the vanishing of higher dimensional cohomology groups.

Theorem 3. If $H^{n+2}(M, \mathbf{C})=0$ and $\operatorname{dim} M / \overline{M^{2}}=m \geq n \geq 0$, then there exists a neighbourhood $\mathcal{U}$ of the corresponding point $\omega \in \mathcal{M}_{A}$, in the metric topology, that is an m-dimensional analytic polydisc. 
Proof. By Theorem $1 M$ has the $\pi$-property. Next, as in the proof of Theorem 2, we take $\mu \in \Lambda,|\mu|=k \geq 2$ and prove that $G_{\mu}$ annihilates $\operatorname{Ker} \pi$. Recall that $G_{\mu}$ is symmetric.

Consider $\phi \in L^{n+2}(M, \mathbf{C})$ given by the formula

$$
\phi\left(a_{1}, a_{2}, \ldots, a_{n+2}\right)=t_{1}\left(a_{1}\right) \ldots t_{n}\left(a_{n}\right) G_{\mu}\left(a_{n+1} \otimes a_{n+2}\right) .
$$

A routine calculation similar to that in the proof of Theorem 2 shows that $\delta \phi=0$ and hence $\phi \in \mathcal{Z}^{n+2}(M, \mathbf{C})$. Therefore $\phi \in \mathcal{B}^{n+2}(M, \mathbf{C})$, so there exists $S \in L^{n+1}$ $(M, \mathbf{C})$ such that $\phi=\delta S$, that is

$$
\begin{aligned}
t_{1}\left(a_{1}\right) \ldots t_{n}\left(a_{n}\right) G_{\mu}\left(a_{n+1} \otimes a_{n+2}\right) & =S\left(a_{1} a_{2}, a_{3}, \ldots, a_{n+2}\right)-S\left(a_{1}, a_{2} a_{3}, \ldots, a_{n+2}\right)+\cdots \\
& +(-1)^{n+2} S\left(a_{1}, a_{2}, \ldots, a_{n}, a_{n+1} a_{n+2}\right) .
\end{aligned}
$$

Now we take an arbitrary $u \in \operatorname{Ker} \pi$ and represent $u=\sum_{i=1}^{\infty} a_{i} \otimes b_{i}$, where $\sum_{i=1}^{\infty}\left\|a_{i}\right\|\left\|b_{i}\right\|<\infty$ and $\sum_{i=1}^{\infty} a_{i} b_{i}=0$.

Then apply the functional $t_{1} \otimes \cdots \otimes t_{n} \otimes G_{\mu}$ to all elementary tensors obtained from $h_{1} \otimes \cdots \otimes h_{n} \otimes a_{i} \otimes b_{i}$ by permutations, in which $a_{i}$ remains to the left of $b_{i}$. After this add all the equations together multiplying by the signs of the corresponding permutation. Finally, take the summation over $i$.

On the one hand we obtain $\sum_{i=1}^{\infty} G_{\mu}\left(a_{i} \otimes b_{i}\right)=G_{\mu}(u)$, because the other terms are either zero or cancel in pairs, by the symmetry of $G_{\mu}$. On the other hand, using $(* *)$ we get sums of terms of two kinds:

(i) $\sum_{i=1}^{\infty} S\left(h_{k_{1}}, \ldots, a_{i} b_{i}, \ldots, h_{k_{n}}\right)$, these sums are zero as $\sum_{i=1}^{\infty} a_{i} b_{i}=0$;

(ii) $S\left(h_{k_{1}}, \ldots, h_{k_{m}} h_{k_{m+1}}, \ldots, a_{i}, \ldots, b_{i}, \ldots\right)$ or $S\left(h_{k_{1}}, \ldots, h_{k_{m}} a_{i}, \ldots, b_{i}, \ldots\right)$, all such terms occur twice and cancel pairwise.

Thus we have shown that $G_{\mu}(u)=0$, and using Proposition 2 and Theorem R, we complete the proof.

Note 1 . In view of Proposition 3 the number $m$ in Theorem 3 actually can only be either $n$ or $n+1$.

Note 2. Theorem 3 seems to be stronger than the main result of the first author's paper [10]. But in that paper an analytic polydisc is obtained in a Gelfand neighbourhood. This stronger conclusion cannot be obtained from Theorem $\mathrm{R}$ without some additional proof, as well as the criterion for projectivity of $M$ in [8].

5. Homology groups and analytic polydises In this final section we show that one can conclude the existence of analytic polydiscs from the vanishing of certain homology groups and assumptions about the dimension of $M / \overline{M^{2}}$. These results should be compared with those of the last section and also results in [10]. In that paper the first author obtained Gelfand neighbourhoods under assumptions about the homological dimension of the maximal ideal. The dimension assumption in [10] is analogous to the case $H_{n+2}(M, \mathbf{C})=0$ and $\operatorname{dim} M / \overline{M^{2}}=n+1$ presented here.

We begin with a lemma. We define two operators which give rise to $n$-cycles. Let $T: \hat{\otimes}^{n} M \rightarrow \hat{\otimes}^{n} M$ be defined by 


$$
T\left(a_{1} \otimes a_{2} \otimes \cdots \otimes a_{n}\right)=\sum_{\pi \in S_{n}} \epsilon_{\pi} a_{\pi(1)} \otimes a_{\pi(2)} \otimes \cdots a_{\pi(n)},
$$

and let $S: \hat{\otimes}^{n+2} M \rightarrow \hat{\otimes}^{n+2} M$ be defined by

$$
S\left(a_{1} \otimes a_{2} \otimes \cdots \otimes a_{n+2}\right)=\sum_{\pi \in S_{n+2}, \pi^{-1}(n+1)<\pi^{-1}(n+2)} \epsilon_{\pi} a_{\pi(1)} \otimes a_{\pi(2)} \otimes \cdots a_{\pi(n+2)} .
$$

Lemma 2. Let $S$ and $T$ be as above and let $\sum_{j=1}^{\infty} a_{n+1}^{(j)} \otimes a_{n+2}^{(j)}$ be an element of Ker $\pi$ then $T\left(a_{1} \otimes a_{2} \otimes \cdots \otimes a_{n}\right)$ and $\sum_{j=1}^{\infty} S\left(a_{1} \otimes a_{2} \otimes \cdots \otimes a_{n} \otimes a_{n+1}^{(j)} \otimes a_{n+2}^{(j)}\right)$ are cycles.

Proof.

$$
d\left(T\left(a_{1} \otimes a_{2} \otimes \cdots \otimes a_{n}\right)\right)=\sum_{k=1}^{n-1}(-1)^{k-1} \sum_{\pi \in S_{n}} \epsilon_{\pi} a_{\pi(1)} \otimes \cdots \otimes a_{\pi(k)} a_{\pi(k+1)} \otimes \cdots a_{\pi(n)} .
$$

Clearly interchanging $k$ and $k+1$ gives terms which cancel in pairs. Similarly

$$
\begin{aligned}
& d\left(\sum_{j=1}^{\infty} S\left(a_{1} \otimes a_{2} \otimes \cdots \otimes a_{n} \otimes a_{n+1}^{(j)} \otimes a_{n+2}^{(j)}\right)\right)= \\
& \sum_{j=1}^{\infty} \sum_{k=1}^{n+1}(-1)^{k-1} \sum_{\pi \in S_{n+2}, \pi(n+1)<\pi(n+2)} \epsilon_{\pi} a_{\pi(1)}^{(j)} \otimes \cdots \otimes a_{\pi(k)}^{(j)} a_{\pi(k+1)}^{(j)} \otimes \cdots a_{\pi(n+2)}^{(j)},
\end{aligned}
$$

where $a_{k}^{(j)}=a_{k}$ for $1 \leq k \leq n$. Clearly interchanging $k$ and $k+1$ gives terms which cancel in pairs. This is allowable except when $\pi(k)=n+1$ and $\pi(k+1)=n+2$. In this case the terms cancel when they are summed over $j$.

Theorem 4. Let $H_{n+2}(M, \mathbf{C})=0$, then $\operatorname{dim} M / \overline{M^{2}}<n+2$.

Proof. Assume this is not the case and let $h_{1}, h_{2}, \ldots, h_{n+2}$ be elements of $M$ which are linearly independent in the quotient $M / \bar{M}^{2}$. Let $t_{1}, \ldots, t_{n+2}$ be dual functions which vanish on $\overline{M^{2}}$ and such that $t_{i}\left(h_{j}\right)=\delta_{i j}$. By Lemma $2 T\left(h_{1} \otimes \cdots \otimes h_{n+2}\right)$ is a cycle. If $H_{n+2}(M, \mathbf{C})=0$, it must also be a boundary, thus we have

$$
\begin{aligned}
T\left(h_{1} \otimes \cdots\right. & \left.\otimes h_{n+2}\right)=\sum_{j=1}^{\infty} d\left(a_{1}^{(j)} \otimes a_{2}^{(j)} \otimes \cdots \otimes a_{n+3}^{(j)}\right) \\
& =\sum_{j=1}^{\infty} \sum_{k=1}^{n+2}(-1)^{k-1} a_{1}^{(j)} \otimes \cdots a_{k}^{(j)} a_{k+1}^{(j)} \otimes \cdots \otimes a_{n+3}^{(j)}
\end{aligned}
$$

If we now apply $t_{1} \otimes t_{2} \otimes \cdots t_{n+2}$ to both sides, the left hand side equals 1 , whereas the right hand side equals 0 . This contradiction proves the result. 
Theorem 5. Let $H_{n+2}(M, \mathbf{C})=0$ and $\operatorname{dim} M / \overline{M^{2}} \geq n$, then $H_{2}^{s}(M, \mathbf{C})=0$.

Proof. Choose $h_{1}, h_{2}, \ldots, h_{n}$ and $t_{1}, \ldots, t_{n}$ as above. We denote an arbitrary element of Ker $\pi=\mathcal{Z}_{2}$ by $\sum_{j=1}^{\infty} h_{n+1}^{(j)} \otimes h_{n+2}^{(j)}$. By Lemma 2 we have

$$
S\left(\sum_{j=1}^{\infty} h_{1} \otimes \cdots \otimes h_{n+1}^{(j)} \otimes h_{n+2}^{(j)}\right)
$$

is an $(n+2)$-cycle. As $H_{n+2}(M, \mathbf{C})=0$, it is also a boundary, hence equals

$$
d\left(\sum_{j=1}^{\infty} a_{1}^{(j)} \otimes a_{2}^{(j)} \otimes \cdots \otimes a_{n+3}^{(j)}\right)=\sum_{j=1}^{\infty} \sum_{k=1}^{n+2}(-1)^{k-1} a_{1}^{(j)} \otimes \cdots a_{k}^{(j)} a_{k+1}^{(j)} \otimes \cdots \otimes a_{n+3}^{(j)}
$$

as above. We now apply the map $t_{1} \otimes t_{2} \otimes \cdots \otimes t_{n} \otimes I_{M} \otimes I_{M}$ to each side of the identity (and use $\otimes^{n} \mathbf{C} \hat{\otimes} M \hat{\otimes} M \cong M \hat{\otimes} M$ ) to obtain

$$
\sum_{j=1}^{\infty}\left[h_{n+1}^{(j)} \otimes h_{n+2}^{(j)}+\sum_{k=1}^{n}\left[-t_{k}\left(h_{n+1}^{(j)}\right) h_{k} \otimes h_{n+2}^{(j)}+t_{k}\left(h_{n+1}^{(j)}\right) h_{n+2}^{(j)} \otimes h_{k}\right]\right]
$$

on the left hand side. This is the original 2-cycle plus an anti-symmetric 2-chain. Note that anti-symmetric 2-chains are always 2-cycles as the algebra is commutative. On the right hand side we obtain

$$
(-1)^{n} \sum_{j=1}^{\infty} t_{1}\left(a_{1}^{(j)}\right) \cdots t_{n}\left(a_{n}^{(j)}\right)\left[a_{n+1}^{(j)} a_{n+2}^{(j)} \otimes a_{n+3}^{(j)}-a_{n+1}^{(j)} \otimes a_{n+2}^{(j)} a_{n+3}^{(j)}\right]
$$

which is a 2-boundary. This shows that $\mathcal{Z}_{2}=C_{2}^{a}+\mathcal{B}_{2}$. Now we can symmetrize this equation, using the $o p$ operator used in the definition of the symmetric homology groups. Hence $\mathcal{Z}_{2}^{s}=\mathcal{B}_{2}^{s}$ i.e., $H_{2}^{s}(M, \mathbf{C})=0$.

Theorem 6. Let $H_{n+1}(M, \mathbf{C})=0$ and $\operatorname{dim} M / \overline{M^{2}} \geq n$, then $M$ has the $\pi$-property.

Proof. We let $c$ be some element of $\overline{M^{2}}$ and choose $h_{1}, h_{2}, \ldots, h_{n}$ and $t_{1}, \ldots, t_{n}$ as above. Then by Lemma $2 T\left(h_{1} \otimes \cdots \otimes h_{n} \otimes c\right)$ is a cycle and as $H_{n+1}(M, \mathbf{C})=0$ it is also a boundary. Hence

$$
\begin{aligned}
T\left(h_{1} \otimes \cdots \otimes h_{n} \otimes c\right) & =d\left(\sum_{j=1}^{\infty} a_{1}^{(j)} \otimes a_{2}^{(j)} \otimes \cdots \otimes a_{n+2}^{(j)}\right) \\
& =\sum_{j=1}^{\infty} \sum_{k=1}^{n+1} a_{1}^{(j)} \otimes \cdots a_{k}^{(j)} a_{k+1}^{(j)} \otimes \cdots \otimes a_{n+2}^{(j)}
\end{aligned}
$$

We apply $t_{1} \otimes \cdots \otimes t_{n} \otimes I_{M}$ to the resulting equality and obtain 


$$
c=\sum_{j=1}^{\infty} t_{1}\left(a_{1}^{(j)}\right) \cdots t_{n}\left(a_{n}^{(j)}\right) a_{n+1}^{(j)} a_{n+2}^{(j)} .
$$

This shows that $c$ is in $\operatorname{Im} \pi$.

Finally we are in a position to prove the result on analytic structure.

Theorem 7. If $H_{n+1}(M, \mathbf{C})=0$ and $\operatorname{dim} M / \overline{M^{2}}=n$, then $H_{s}^{2}(M, \mathbf{C})=0$ and there exists a neighbourhood $\mathcal{U}$ of the corresponding point $\omega \in \mathcal{M}_{A}$, in the metric topology, that is an n-dimensional analytic polydisc.

Proof. By Theorem 2 it suffices to show that $H_{s}^{2}(M, \mathbf{C})=0$. Let $\phi$ be any symmetric 2-cocycle. Then $\phi$ vanishes on every anti-symmetric 2 -cycle, by symmetry. It also vanishes on every symmetric 2-cycle as these are all boundaries (of symmetric 3cocycles) by Theorem 5. Thus $\phi$ vanishes on Ker $\pi$. We now use the $\pi$-property, this shows that $\phi=\psi \circ \pi$ for some continuous functional $\psi$ on $M$. Thus $\phi=\delta \psi$ and we are done.

ACKnOwLEDGements. The first author was partially supported by the Soros ISF foundation and by the RFFI grant No. 96-01-1036.

\section{REFERENCES}

1. W. G. Bade, H. G. Dales and Z. A. Lykova, Algebraic and strong splittings of extensions of Banach algebras, preprint.

2. A. Browder, Point derivations and analytic structure in the spectrum of a Banach algebra, J. Functional Anal. 7 (1971), 156-164.

3. P. Dixon, Non-separable Banach algebras whose squares are pathological, J. Functional Anal. 26 (1977), 190-200.

4. A. Grothendieck, Produits tensorielles topologiques et espaces nucleaires. Mem. Amer. Math. Soc. 16 (1955), 3-191.

5. A. Ya. Helemskñ, A certain class of flat Banach modules over Banach algebras and its applications, Vest. Mosk. Univ. ser. mat. mekh 27:1 (1972), 29-36.

6. A. Ya. Helemskĩ, Annihilator extensions of commutative Banach algebras, Izv. Akad. Nauk SSSR ser. mat. 29 (1965), 945-956. $3-121$.

7. B. E. Johnson, Cohomology in Banach algebras. Mem. Amer. Math. Soc. 127 (1972),

8. L. I. Pugach, On flat ideals of Banach algebras, Vest. Mosk. Univ. ser. mat. mehk. 1 (1981), 59-63 [Moscow Univ. Math. Bull. 36 (1981), 70-74].

9. L. I. Pugach, Projective and flat ideals of function algebras and their connection with analytic structure, Mat. Zametki 31 (1982), 239-245 [Math. Notes 31 (1982), 114-117].

10. L. I. Pugach, Homological properties of function algebras and analytic polydisks in their maximal ideal space, Rev. Roumaine Math. Pure and Appl. 31 (1986) 347-356 [Selecta Mathematica Sovietica 8 (1989), 259-269].

11. T. T. Read, The powers of a maximal ideal in a Banach algebra and analytic structure, Trans. Amer. Math. Soc. 161 (1971), 235-248. 